\title{
Cortical neurons are a prominent source of the proinflammatory cytokine osteopontin in HIV-associated neurocognitive disorders
}

\author{
Katie Silva • Calixto Hope-Lucas • Tyesha White • \\ Tai-Kyung Hairston • Tatenda Rameau • Amanda Brown
}

Received: 25 September 2014 /Revised: 30 December 2014 / Accepted: 9 January 2015 / Published online: 31 January 2015

(C) The Author(s) 2015. This article is published with open access at Springerlink.com

\begin{abstract}
The proinflammatory cytokine osteopontin (OPN) is elevated in the cerebrospinal fluid (CSF) in individuals with HIV-associated neurocognitive disorders (HAND) and remains so in those on suppressive antiretroviral therapy. To understand the pathophysiological significance of elevated OPN in the CNS, we sought to determine the cellular source of this cytokine. As HIV-1 replicates productively in macrophages/microglia, we tested whether these cells are the predominant producers of OPN in the brain. Stringent patient selection criteria, which excluded brain tissues from those with evidence of drug abuse and dependence, were used. Uninfected normal controls, amyotrophic lateral sclerosis (ALS), HIV+ asymptomatic neurocognitive impairment (ANI), and HIV+ mild neurocognitive disorder (MND)/HIVassociated dementia (HAD) groups were included. Doublelabel immunohistochemistry for CNS cells and OPN was used to quantify OPN expression in astrocytes, macrophages $/ \mathrm{mi}$ croglia, and neurons. While resident macrophages/microglia expressed OPN, astrocytes and unexpectedly neurons were also a major source of OPN. OPN levels in ionized $\mathrm{Ca}^{2+}$ binding adapter 1 (Iba1)/allograft inflammatory factor-1 (AIF-1)+ microglia in HIV+ ANI and MND/HAD exceeded those of HIV-negative controls and were comparable to expression seen in ALS. Moreover, in neurons, OPN was expressed at the highest levels in the HIV+ ANI group. These findings suggest that while infiltrating HIV-infected macrophages are most likely the initial source of OPN, resident CNS cells become activated and also express this inflammatory cytokine at significant levels. Moreover, as OPN
\end{abstract}

K. Silva • C. Hope-Lucas • T. White · T.-K. Hairston • T. Rameau •

A. Brown $(\square)$

Department of Neurology, Johns Hopkins University School of

Medicine, 600 North Wolfe Street/Meyer 6-181,

Baltimore, MD 21287-7131, USA

e-mail: abrown76@jhmi.edu levels are elevated compared to uninfected individuals and increases with the severity of impairment, it appears that the expression of OPN is persistent and sustained within the brain parenchyma in those that progress to HAND.

Keywords Neuroinflammation · Neurodegeneration · CD68 - Iba-1 · AIF-1 · Inflammation · Macrophage . Microglia $\cdot$ Astrocytes $\cdot$ Systemic inflammation

\section{Introduction}

Trafficking of HIV-infected macrophages and activated uninfected monocytes/macrophages into the brain occurs early in the course of viral infection (Budka et al. 1987; Davis et al. 1992; Glass et al. 1995; Koenig et al. 1986) and initiates the seeding of resident brain macrophages, microglia, and astrocytes with HIV/SIV DNA (Clements et al. 2002). The local immune response in the brain results in an inflammatory milieu that exacerbates the release of substances including glutamate leading, by mechanisms that remain to be fully elucidated, to neurotoxicity, neuronal dysfunction, and cognitive impairment collectively known as HIV-associated neurocognitive disorders (HAND) (Kraft-Terry et al. 2010; Vázquez-Santiago et al. 2014). HAND is assessed through a battery of neuropsychological tests and encompasses three levels of impairment: asymptomatic neurocognitive impairment (ANI), HIV-associated mild neurocognitive disorder (MND), and HIV-associated dementia (HAD) (Antinori et al. 2007). The prevalence of HIV-associated neurocognitive disorders and particularly the milder forms has increased, while the incidence of severe debilitating dementia has dramatically decreased (McArthur et al. 2010). This latter finding reflects the high efficacy of suppressing HIV replication, which results in significant reductions in viral load in the 
blood and cerebrospinal fluid (CSF) with combination antiretroviral therapy (ART) that target two or more steps in the viral life cycle. However, in up to $50 \%$ of HIV-infected individuals on ART, cognitive dysfunction remains a significant problem (Heaton et al. 2010; Heaton et al. 2011), and currently, no adjunctive neuroprotective therapies have yet reached routine clinic practice (Meulendyke et al. 2014).

Several potential explanations have been proposed to explain the continued impairment including the inefficient penetration of ART into the brain parenchyma that would permit low-level ongoing viral replication at this site, toxicity of ART and its metabolites (Tovar-y-Romo et al. 2012), and comorbidities including aging and cardiovascular disease could all potentially contribute to the continued dysfunction seen in HIV-infected individuals (Deeks et al. 2013). Additionally, markers of immune activation remain elevated in the periphery and CNS in even the most well-suppressed HIV-infected ART-treated individuals, suggesting the continued stimulation and hyperactivity of the immune system to antigens from several anatomical locations including the intestine (Brenchley et al. 2006; Spudich et al. 2011). Inflammation in the brain in response to injury or invasion by microorganisms can play either beneficial and/or neuropathogenic roles depending on the intensity and duration of the response (Katsumoto et al. 2014).

Osteopontin (OPN), a proinflammatory cytokine of the innate immune system, was first reported to be increased in the brains of SIVE-infected macaques and later in the plasma, CSF, and brains of HIV-infected individuals with cognitive impairment (Brown et al. 2011; Burdo et al. 2008). A recent study suggests that OPN remains elevated in plasma in those on ART (Burdo et al. 2011). Interestingly, OPN is elevated in other neurodegenerative disorders including multiple sclerosis (MS), Alzheimer's disease, Parkinson's disease, and frontotemporal dementia (Braitch et al. 2008; Chabas et al. 2001; Comabella et al. 2005; Comi et al. 2010; Iczkiewicz et al. 2006; Maetzler et al. 2007; Mattsson et al. 2008; Wirths et al. 2010). Only in the case of MS has the role of OPN been explored where in mouse models of the disorder, OPN has been shown to facilitate the survival of anti-myelin autoreactive T cells (Hur et al. 2006; Jansson et al. 2002). We have shown that OPN is significantly elevated in HIV-infected macrophages (Brown et al. 2011). Inhibition of OPN in macrophages with siRNA inhibits HIV replication $50 \%$ suggesting that OPN stimulates viral replication likely through a NF-KB-dependent mechanism acting via OPN receptors, thus forming a feedback loop which promotes continued cellular activation and viral spread (Brown et al. 2011; Eger et al. 2014). Because of their central role in the development of HIV-associated cognitive impairment, and to gain an understanding of the impact of activated HIV-infected macrophages on the expression of OPN in the context of the central nervous system (CNS), we used double-label immunochemistry for cells of the human brain and OPN to test the hypothesis that resident macrophages/microglia are the major sources of this proinflammatory cytokine associated with HAND. Four groups of brain tissue were compared including normal, HIV-uninfected controls, HIV-positive with ANI, HIVpositive MND/HAD, and a group with amyotrophic lateral sclerosis (ALS). ALS is a progressive motor neuron degenerative disorder, in which macrophage and microglial activation is now thought to be involved in the pathological progression of the disease. Moreover, recent studies suggest that microglial activation may be associated with deficits in executive function (Brettschneider et al. 2012a; Brites and Vaz 2014). As such, ALS samples were included as a "neurodegenerative other" control. While astrocytes and resident macrophage/microglia express OPN, to our surprise we found that in addition, neurons significantly contribute to the pool of OPN found in the brain in individuals with HAND. These findings suggest that while HIV-infected macrophages stimulate the initial increase of OPN in the brain, resident macrophage/microglia, astrocytes, as well as neurons overexpress this cytokine. As all of these cells express the receptors for OPN (Ailane et al. 2013; Akiyama et al. 1993), our findings suggest that microglial, astrocytic, and neuronal activity and function can potentially be directly altered by OPN through paracrine regulatory mechanisms as a result.

\section{Results}

Patient samples and clinical characteristics

Microarray analyses on the brains of SIV-infected macaques identified numerous genes including osteopontin (OPN) that were differentially regulated (Roberts et al. 2003). Further investigations found that OPN was significantly elevated in the plasma of rhesus macaques and CSF and brain in individuals with HAND (Brown et al. 2011; Burdo et al. 2008; Eger et al. 2014). Burdo et al. proposed HIV-infected and/or activated macrophage trafficking into the brain releases OPN, which has chemotactic and antiapoptotic signaling potential, and serves in a feedback loop fueling the establishment of HIV in the brain as well as the cellular activation and inflammation at this site (Burdo et al. 2007). To better understand the potential neuropathogenic role for elevated OPN in HAND, in this study, we tested the hypothesis that activated macrophages/resident microglia are the cellular sources of OPN in the brain. Stringent patient sample criteria were used including the exclusion of the drugs of abuse cocaine, cannabis, opiate, and methadone. In addition, toxicology reports were examined to exclude drug abuse and dependence. We used double-label immunohistochemistry and antibodies to CD68 and Iba-1/AIF-1 to specifically identify macrophage/ microglia in brain tissue from uninfected controls, HIV+ 
ANI, HIV+ MND/HAD, and a group with a well-known neurodegenerative disease affecting motor neurons, amyotrophic lateral sclerosis, ALS. Moreover, as astrocytes and neurons have been reported to express increased levels of OPN in models of brain injury and neurodegeneration, we also used anti-glial fibrillary acidic protein (GFAP) and anti-neuronal nuclei $(\mathrm{NeuN})$ antibodies to determine whether this proinflammatory cytokine is elevated in these cell types. The demographics and clinical parameters of the patients used are shown in Table 1. There were no significant differences in age of the subjects in each group (normal, 47.7 \pm 7.35; ALS, 50.4 7.37, HIV ANI 43.4 \pm 7.89 ; HIV $\mathrm{NMD} / \mathrm{HAD}, 40.5 \pm 10.5$ ).
Osteopontin is significantly elevated in GFAP-reactive astrocytes from $\mathrm{HIV}+\mathrm{MND} / \mathrm{HAD}$ cases compared to ALS samples

GFAP reactivity was highest in the upper cortical layers in most samples and in some cases, positively stained cells were visible throughout all cortical layers (Fig. 1). In the HIV+ MND/HAD group, astrocytic cell bodies devoid of processes often predominated (Fig. 1, cases 19 and 20). Compared to the normal control group $(0.612 \pm 0.255, n=9)$, OPN levels did not differ significantly with the other groups $(\mathrm{HIV}+\mathrm{NC}$, $0.576 \pm 0.212, n=5$; HIV + impaired, $0.682 \pm 0.254, n=5$; ALS, $0.524 \pm 0.207, n=5$ ), although there was a trend of

Table 1 Patient sample demographics

\begin{tabular}{|c|c|c|c|c|c|c|c|c|c|}
\hline Patient ID & Age & Sex & Race & HIV status & Neurocog diagnosis & Plasma VL & CSF VL & CD4 & ARV \\
\hline Case 1 & 47 & $\mathrm{~F}$ & Black & Neg & Normal & NA & NA & NA & NA \\
\hline Case 2 & 44 & $\mathrm{~F}$ & White & $\mathrm{Neg}$ & Normal & NA & NA & NA & NA \\
\hline Case 3 & 51 & M & White/Hispanic & $\mathrm{Neg}$ & Normal & NA & NA & NA & NA \\
\hline Case 4 & 49 & M & White & $\mathrm{Neg}$ & Normal & NA & NA & NA & NA \\
\hline Case 5 & 46 & M & White & $\mathrm{Neg}$ & Normal & NA & NA & NA & NA \\
\hline Case 6 & 39 & M & Hispanic & Pos & ANI & 570 & $<50$ & 79 & Yes \\
\hline Case 7 & 43 & M & White & Pos & ANI & 249 & 19 & 7 & Yes \\
\hline Case 8 & 39 & M & White & Pos & MND & 72,125 & 70 & 1 & Yes \\
\hline Case 9 & 33 & M & Hispanic & Pos & MND & 400 & NT & 11 & Yes \\
\hline Case 10 & 35 & M & White & Pos & MND/HAD (HIVE) & 2827 & 78 & 211 & Yes \\
\hline Case 11 & 37 & M & White & Pos & MND & 178 & 5968 & 85 & Yes \\
\hline Case 12 & 31 & M & White & Pos & MND & 483,758 & 148 & 10 & Yes \\
\hline Case 13 & 57 & M & Asian & Pos & HAD & 40,133 & 2747 & 299 & Yes \\
\hline Case 14 & 30 & $\mathrm{~F}$ & Hispanic & Neg & Normal & NA & NA & NA & NA \\
\hline Case 15 & 48 & $\mathrm{~F}$ & Hispanic & Neg & Normal & NA & NA & NA & NA \\
\hline Case 16 & 50 & M & Hispanic & Neg & Normal & NA & NA & NA & NA \\
\hline Case 17 & 56 & $\mathrm{~F}$ & White & Neg & NA & NA & NA & NA & NA \\
\hline Case 18 & 57 & M & Black & Pos & MND & 63,953 & NT & 194 & Yes \\
\hline Case 19 & 34 & M & White & Pos & ANI & $>750,000$ & NT & 9 & Yes \\
\hline Case 20 & 46 & M & White & Pos & ANI & $<50$ & $<50$ & 233 & Yes \\
\hline Case 21 & 45 & M & Hispanic & Pos & $\mathrm{MND} / \mathrm{HAD}$ & 628 & 376 & 28 & Yes \\
\hline Case 22 & 31 & $\mathrm{~F}$ & Hispanic & Pos & $\mathrm{MND} / \mathrm{HAD}$ & 744,349 & NT & 4 & Yes \\
\hline Case 23 & 55 & $\mathrm{~F}$ & Black & Pos & ANI & 37,060 & NT & 114 & Yes \\
\hline Case 24 & 56 & $\mathrm{~F}$ & White & $\mathrm{Neg}$ & Normal & NA & NA & NA & NA \\
\hline Case 25 & 38 & M & White & $\mathrm{Neg}$ & ALS & NA & NA & NA & NA \\
\hline Case 26 & 53 & $\mathrm{~F}$ & White & Neg & ALS & NA & NA & NA & NA \\
\hline Case 27 & 50 & M & White & Neg & ALS & NA & NA & NA & NA \\
\hline Case 28 & 54 & M & White & Neg & ALS & NA & NA & NA & NA \\
\hline Case 29 & 57 & $\mathrm{~F}$ & Hispanic & $\mathrm{Neg}$ & ALS & NA & NA & NA & NA \\
\hline
\end{tabular}

Data are from last clinic visit. Age at death

NA not applicable, $N T$ not available, HIV Neg seronegative, HIV Pos seropositive, $V L$ viral load, $A R V$ antiretroviral use, $A N I$ asymptomatic neurocognitive impairment, $M N D$ minor neurocognitive disorder, $H A D \mathrm{HIV}$-associated dementia, $A L S$ amyotrophic lateral sclerosis 
Fig. 1 Osteopontin (OPN) expression in GFAP+ astrocytes. Paraffin-embedded human autopsy tissue from the occipital lobe of cases and controls from the National NeuroAIDS Tissue Consortium (NNTC) was stained sequentially with rabbit polyclonal antisera against GFAP/goat-anti-rabbit-alkaline phosphatase (AP) secondary and developed with permanent FastRed Quanto (red) then reacted with mouse monoclonal antibody to OPN/goat anti-mouse-horse radish peroxidase and developed with 3,3'-diaminobenzidine. a case 14, normal control; b case 29, amyotrophic lateral sclerosis (ALS); c case 19, asymptomatic neurocognitive disorder (ANI); $\mathbf{d}$ case 20, ANI; e case 21, minor neurocognitive disorder/HIVassociated dementia (MND/ $\mathrm{HAD}$ ); $\mathbf{f}$ case 22, MND/HAD
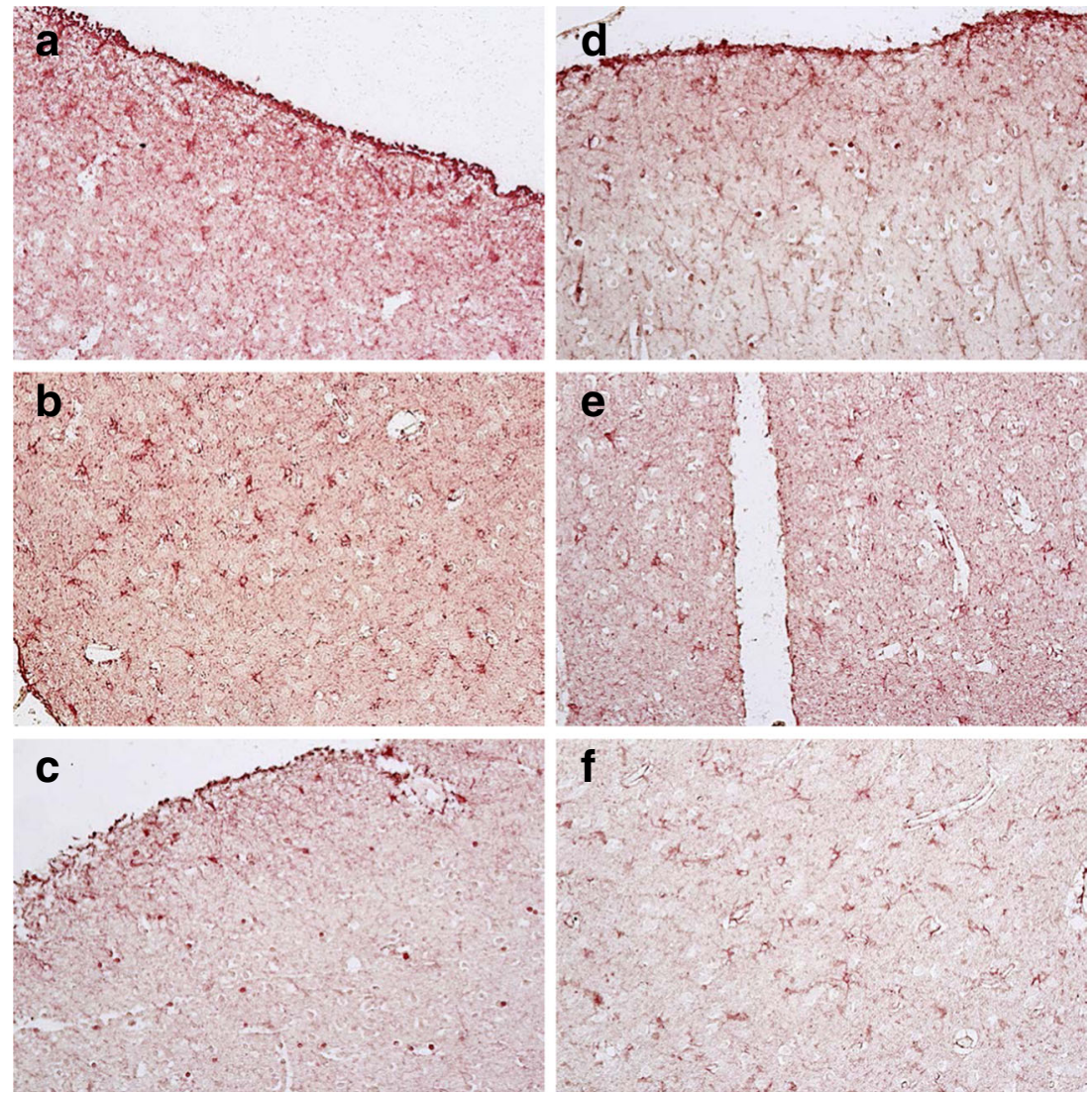

increased OPN in the HIV+ MND/HAD group (Fig. 2a). A significant increase in the level of OPN between the HIV+ $\mathrm{MND} / \mathrm{HAD}$ and the ALS group was detected ( $p=0.011$, Fig. 2). Normalization of GFAP levels to OPN revealed no significant differences in the expression of GFAP among the different groups (Fig. 2b; normal, 2.02 \pm 1.10 ; HIV + ANI,
$2.027 \pm 0.898 ; \mathrm{HIV}+\mathrm{MND} / \mathrm{HAD} 1.722 \pm 0.792 ; \mathrm{ALS}, 2.231 \pm$ 0.948 ). These results suggest that GFAP-reactive astrocytes in HAND express OPN, and while levels between HIV+ NC and $\mathrm{HIV}+\mathrm{MND} / \mathrm{HAD}$ did not differ, astrocytes in the latter case produce significantly higher levels of OPN than what was seen in samples from individuals with ALS.

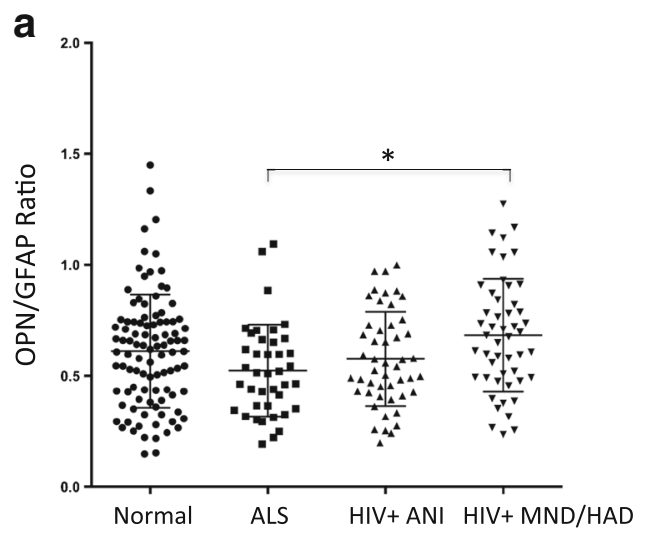

Fig. 2 Osteopontin is significantly elevated in GFAP-reactive astrocytes from HIV+ MND/HAD cases compared to amyotrophic lateral sclerosis (ALS) samples. Ten images were taken at $\times 20$ magnification from all four quadrants of the slides for each case (an equal amount of area), and the intensity (area fraction) of the label for OPN and GFAP were quantified using ImageJ. The results are expressed as the ratio of a OPN to GFAP or b

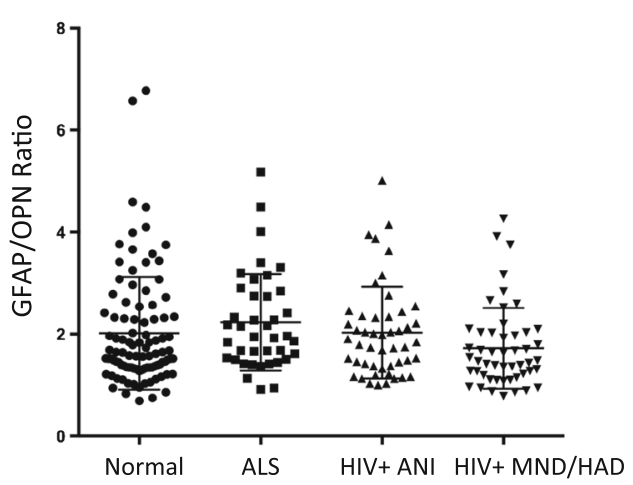

b GFAP to OPN. One-way ANOVA, with Tukey's correction for multiple comparisons and significance of $* p<0.05$, was determined with GraphPad Prism 6. Normal, ALS, amyotrophic lateral sclerosis, HIVinfected cases asymptomatic neurocognitive disorder (HIV+ ANI), and HIV-infected cases with minor neurocognitive disorder/HIV-associated dementia (HIV+ MND/HAD) 
Microglial osteopontin levels are significantly increased in HAND and ALS

Microglia stained for Iba-1/AIF-1 displayed a variety of morphologies including ramified and ameboid shapes with varying degrees of processes (Fig. 3). Unexpectedly, abundant Iba-/AIF-1 staining was seen in several samples from the normal control group (Fig. 3a-b, cases 16 and 4). In addition, there was abundant Iba-1/AIF-1 reactivity in samples from the ALS group with distinctive labeling of microglia with extended ramified shapes (Fig. 3c, case 29). OPN levels in microglia were significantly elevated compared to normal controls $(0.758 \pm 0.376, n=9)$ for ALS $(1.356 \pm 0.848$, $p<0.0001, n=5)$, HIV+ ANI $(1.463 \pm 0.934, p<0.0001, n=$ 5), and HIV+ MND/HAD (1.467 $\pm 0.721, p<0.0001, n=5)$, but there were no differences between the disease groups (Fig. 4a). The level of Iba-I/AIF-1 normalized to OPN intensity was significantly lower in the disease groups (HIV+ ANI, $0.969 \pm 0.570, p<0.0001 ; \mathrm{HIV}+\mathrm{MND} / \mathrm{HAD}, 0.829 \pm 0.358$, $p<0.0001$; ALS, $1.094 \pm 0.782, p<0.0001)$ compared to the normal controls $(1.741 \pm 1.21)$ (Fig. 4b). These results suggest that the amount of OPN expression per microglia was significantly elevated in the diseased groups compared to the normal controls.
Osteopontin levels in cortical neurons are significantly elevated in HIV-infected individuals with ANI compared to HIV-infected cases with cognitive impairment

(MND/HAD) or ALS

In HIV+ and ALS samples, NeuN reactivity was localized in the nucleus as well as in the cytoplasm (Lucas et al. 2014), while OPN staining was restricted to the cytoplasm of neuronal cell bodies (Fig. 5). Unexpectedly, abundant OPN reactive $\mathrm{NeuN}+$ neurons were readily detectable in many of the normal uninfected controls (Fig. 6a). OPN levels were highest in the $\mathrm{HIV}+$ ANI cases $(1.706 \pm 1.045, n=6)$ compared to all other groups (normal, $1.394 \pm 0.748, p=0.005, n=10$; $\mathrm{HIV}+\mathrm{MND} / \mathrm{HAD}, 1.428 \pm 0.589, p=0.023, n=8 ; \mathrm{ALS}$, $1.226 \pm 0.862, p<0.0001, n=5$ ) (Fig. 7). Based on the NeuN/OPN ratio, the ALS group (1.134 \pm 0.632$)$ had significantly more NeuN-reactive neurons compared to the normal $(0.93 \pm 0.59, p=0.018)$, HIV + ANI $(0.844 \pm$ $0.614, p=0.001)$, and HIV + MND/HAD groups $(0.836 \pm$ $0.438, p=0.0002)$, which were similar to each other (Fig. 7b). These results suggest that neurons in cases from HIV+ ANI produce, on average, more OPN than all other groups.
Fig. 3 Osteopontin (OPN) expression in Iba1/AIF-1+ microglia. See staining paradigm as given in figure legend 1 . a case 16, normal; b case 4, normal; c case 29, ALS; d case 23, ANI; e case $12, \mathrm{MND}$; f case $13, \mathrm{HAD}$
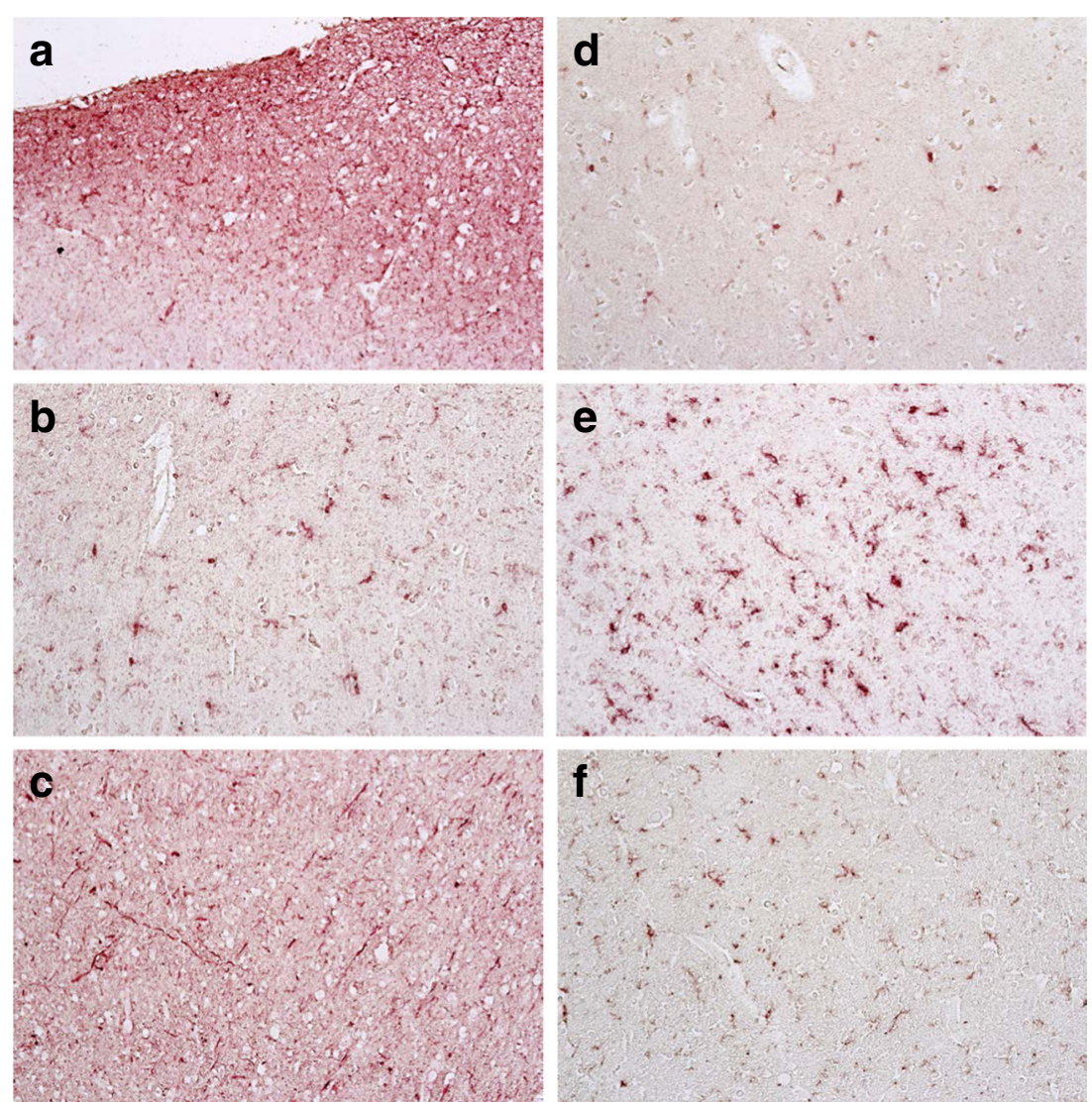


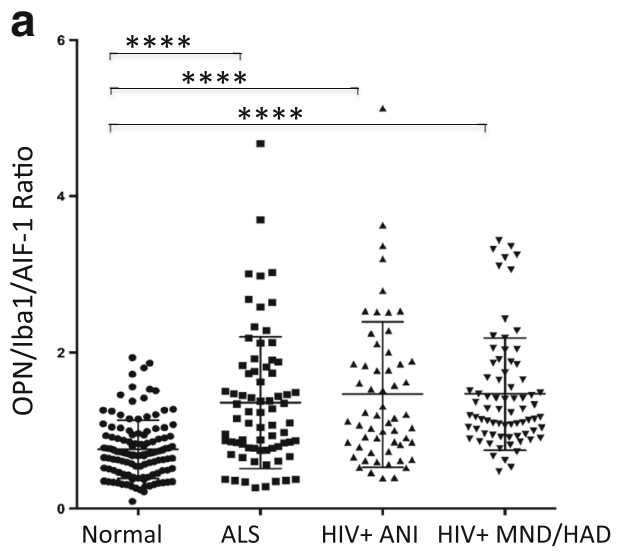

Fig. 4 Microglia-associated osteopontin levels are significantly increased in HAND and ALS. See description of quantification scheme given in figure legend 2. The results are expressed as the ratio of a OPN to Iba1/AIF-1 or b Iba1/AIF-1 to OPN. One-way ANOVA, with Tukey's correction for multiple comparisons and significance of $p<0.05$, was

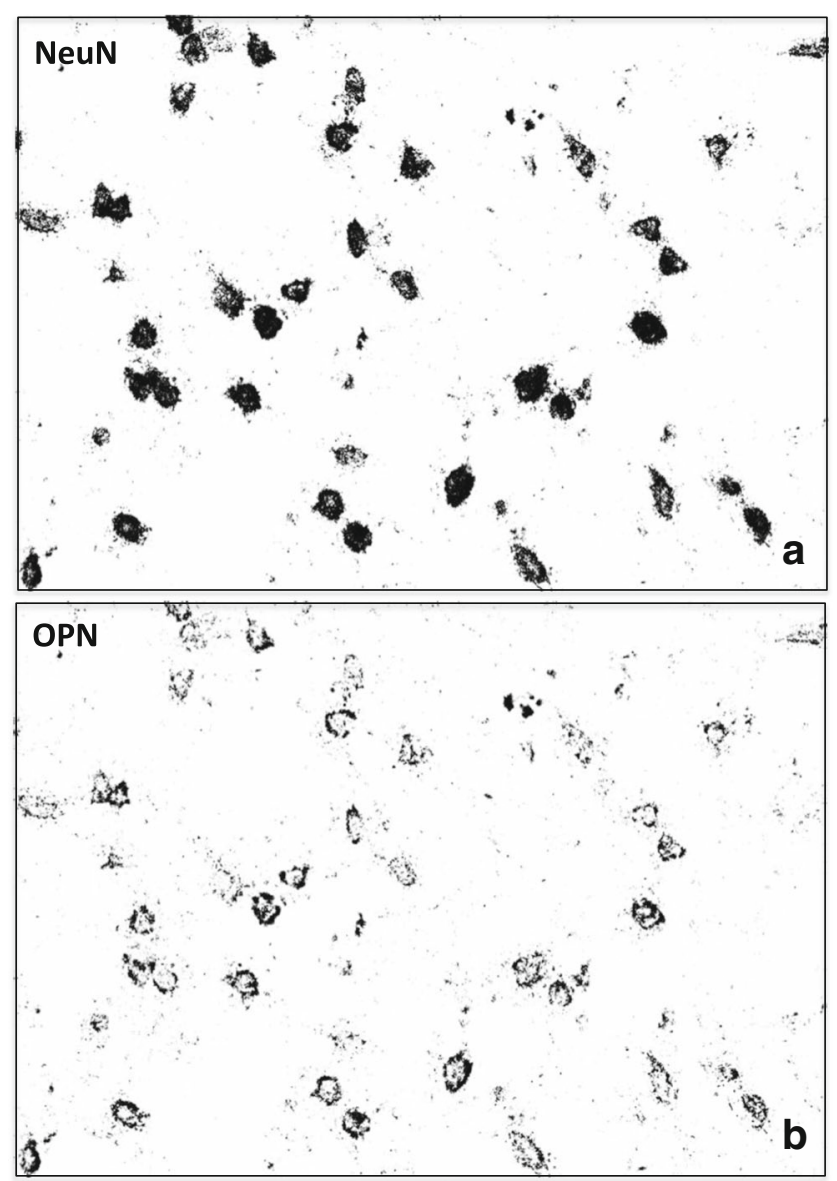

Fig. 5 NeuN reactivity is localized to the nucleus and cytoplasm, while OPN is restricted to the cytoplasm of neuronal cell bodies. Image label intensity for NeuN and OPN reactivity in double-stained sections were analyzed using ImageJ. Top panel, threshold for NeuN positivity. Lower panel, threshold for anti-OPN staining within neurons

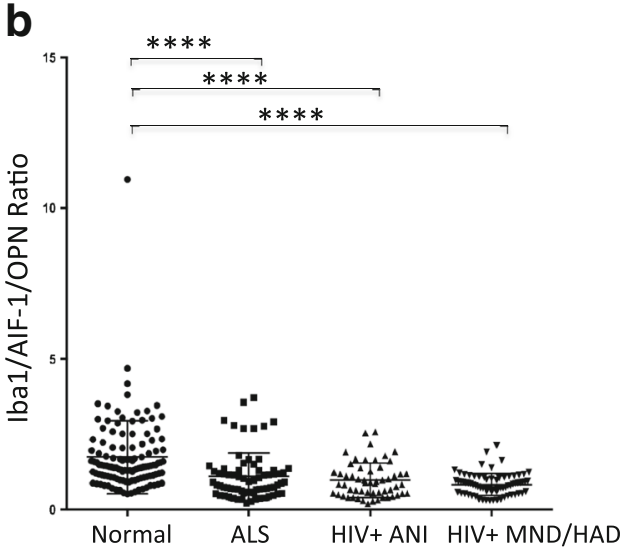

determined with GraphPad Prism 6. Normal, ALS, amyotrophic lateral sclerosis, HIV-infected cases asymptomatic neurocognitive disorder (HIV+ ANI), and HIV-infected cases with minor neurocognitive disorder/HIV-associated dementia (HIV $+\mathrm{MND} / \mathrm{HAD}) ;{ }^{* * * *} p<0.0001$

CD68+ macrophages/microglia are readily detectable in normal control samples

The high level of OPN, GFAP, and Iba-1/AIF-1 expression seen in the normal control group suggested the presence of injury and/or inflammation in the brain. To investigate this possibility, we stained sections with anti-CD68, which detects microglia, as well as perivascular, parenchymal, and resident macrophages (Esiri and McGee 1986), which typically have a finite lifespan in the brain and are not present in large numbers under normal homeostatic conditions (Esiri and O’D McGee 1986; FischerSmith et al. 2004). Strikingly, in 8 out of 10 normal cases, CD68+ macrophages/microglia were readily detectable throughout the cortical layers (Fig. 8). The level of CD68 reactivity was similar to or significantly exceeded that of HIV+ $\mathrm{MND} / \mathrm{HAD}$ samples (case 1 vs. case $21, p=0.0026$; case 1 vs. case 22, $p=0.0101$; case 14 vs. case $21, p=0.0287$; Fig. 9). Table 2 lists the cause of death for samples in the normal control group. In several cases, severe injury to the gastrointestinal tract (Fig. 9, cases 1, 4, and 24) and disorders of the lungs (Fig. 9, cases 2, 5, and 14) displayed the highest levels of CD68 staining, although there were no statistically significant differences between the normal samples with the exception of case $17 \mathrm{vs}$. case 1 ( $p=0.0368)$. These results suggest that the majority of normal controls used in this study, although not infected with HIV, displayed evidence of exposure to an event(s) that promoted a sustained increase of CD68+ macrophages in the brain.

\section{Discussion}

The high level of OPN staining in GFAP+ astrocytes and $\mathrm{NeuN}+$ neurons in the HIV-uninfected control group was very 
Fig. 6 Double-labeled neurons expressing NeuN and OPN. See staining paradigm as given in figure legend 1 . a case 16 , normal; b case 25, ALS; c case

18 , MND; $\mathbf{d}$ case 20 , ANI; e case 21, MND/HAD; $\mathbf{f}$ case $13, \mathrm{HAD}$
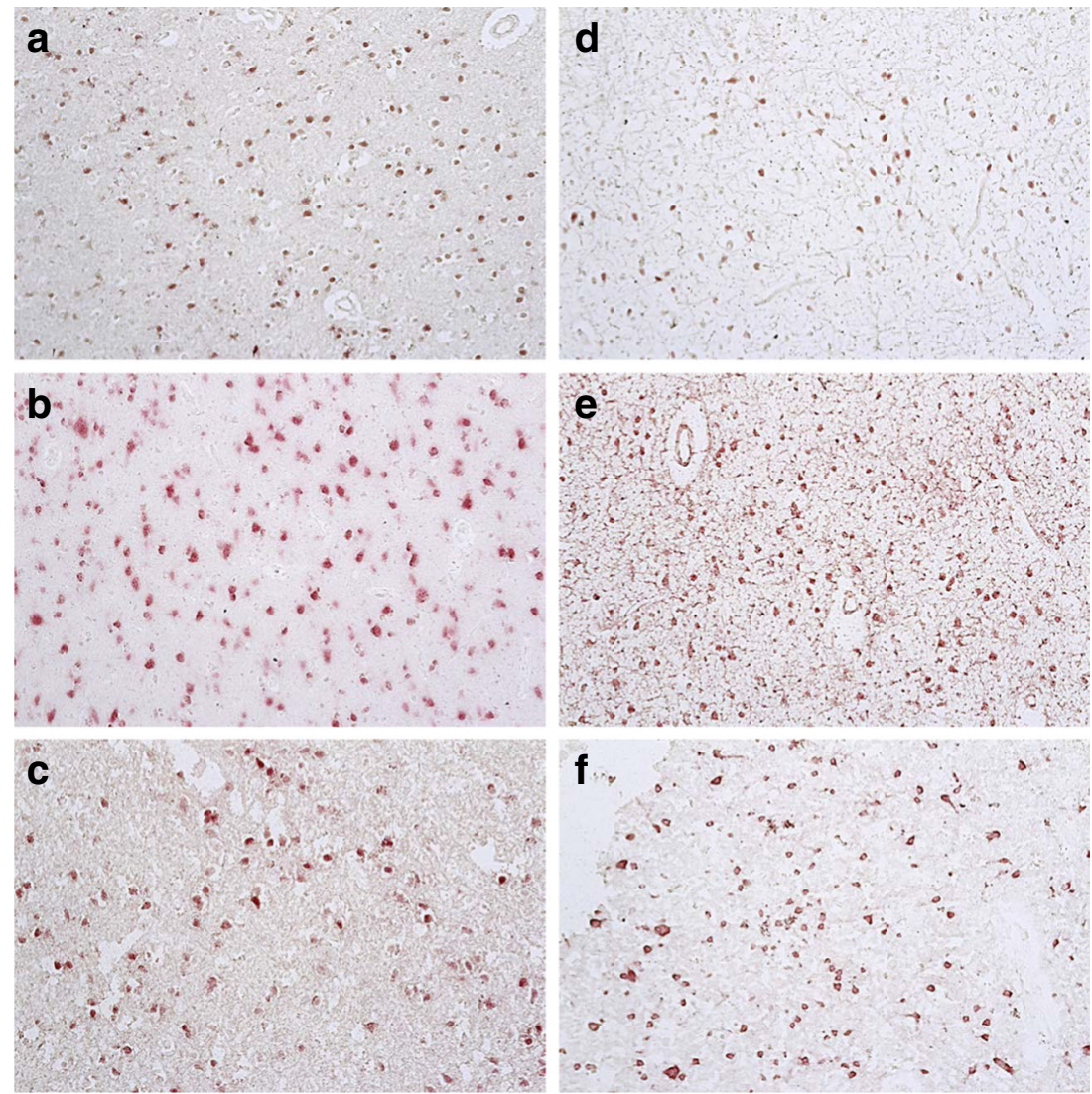

surprising. For these types of studies, normal controls present several limitations: they are less plentiful and more difficult to obtain, and specific clinical data most relevant to the study is often not available. The presence of a high concentration of

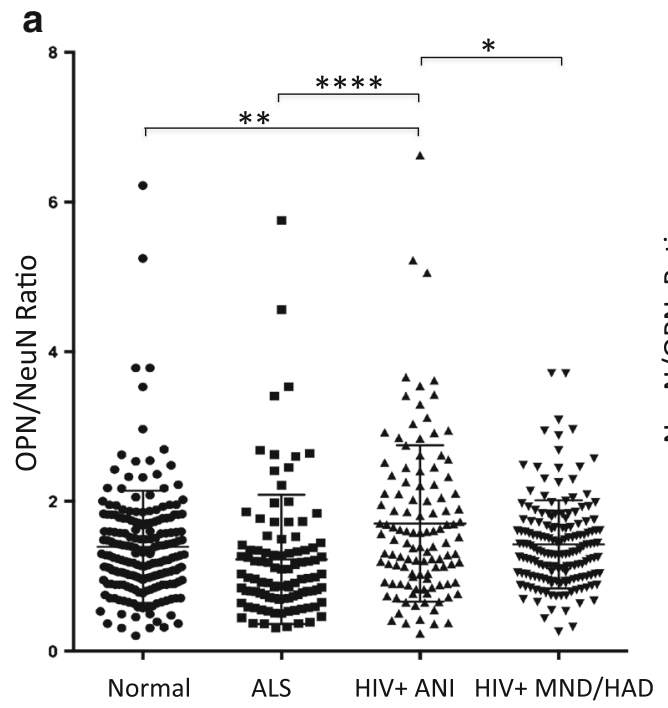

Fig. 7 Osteopontin levels in cortical neurons are significantly elevated in HIV-infected individuals with asymptomatic neurocognitive impairment (ANI) compared to HIV-infected cases with cognitive impairment (MND/ HAD) or ALS. See description of quantification scheme given in figure legend 2. The results are expressed as the ratio of a OPN to NeuN, $* * * * p<0.0001, * * p=0.0052, * p=0.0232$, or b NeuN to OPN,
CD68+ macrophage/microglia strongly suggests that these brains experienced injury and/or pathogenic sequela, which resulted in an inflammatory response. In fact, there is now greater recognition and study of the relationships between

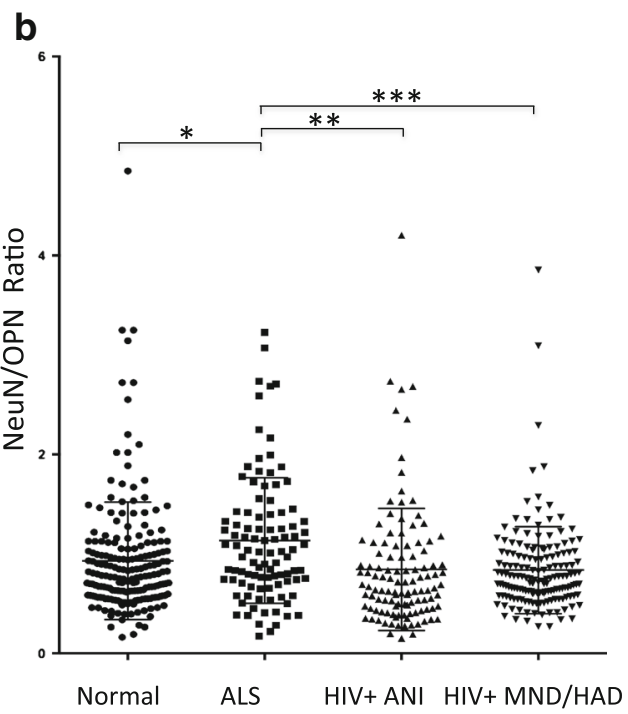

$* * * * p<0.0001, * * * p=0.0002, * * p=0.0011, * p=0.0181$. Normal, ALS, amyotrophic lateral sclerosis, HIV-infected cases asymptomatic neurocognitive disorder (HIV+ ANI), and HIV-infected cases with minor neurocognitive disorder/HIV-associated dementia (HIV+ $\mathrm{MND} / \mathrm{HAD})$ 
Fig. 8 Presence of CD68+ macrophages/microglia in normal and $\mathrm{MND} / \mathrm{HAD}$ groups. See staining paradigm as given in figure legend 1 . a case 14 , normal; $\mathbf{b}$ case 24 , normal; $\mathbf{c}$ case 16 , normal; $\mathbf{d}$ case 1 , normal; $\mathbf{e}$ case 17 , normal; $\mathbf{f}$ case 5 , normal; g case 2, normal; $\mathbf{h}$ case 11 , $\mathrm{MND}$; and $\mathbf{i}$ case 13 , HAD
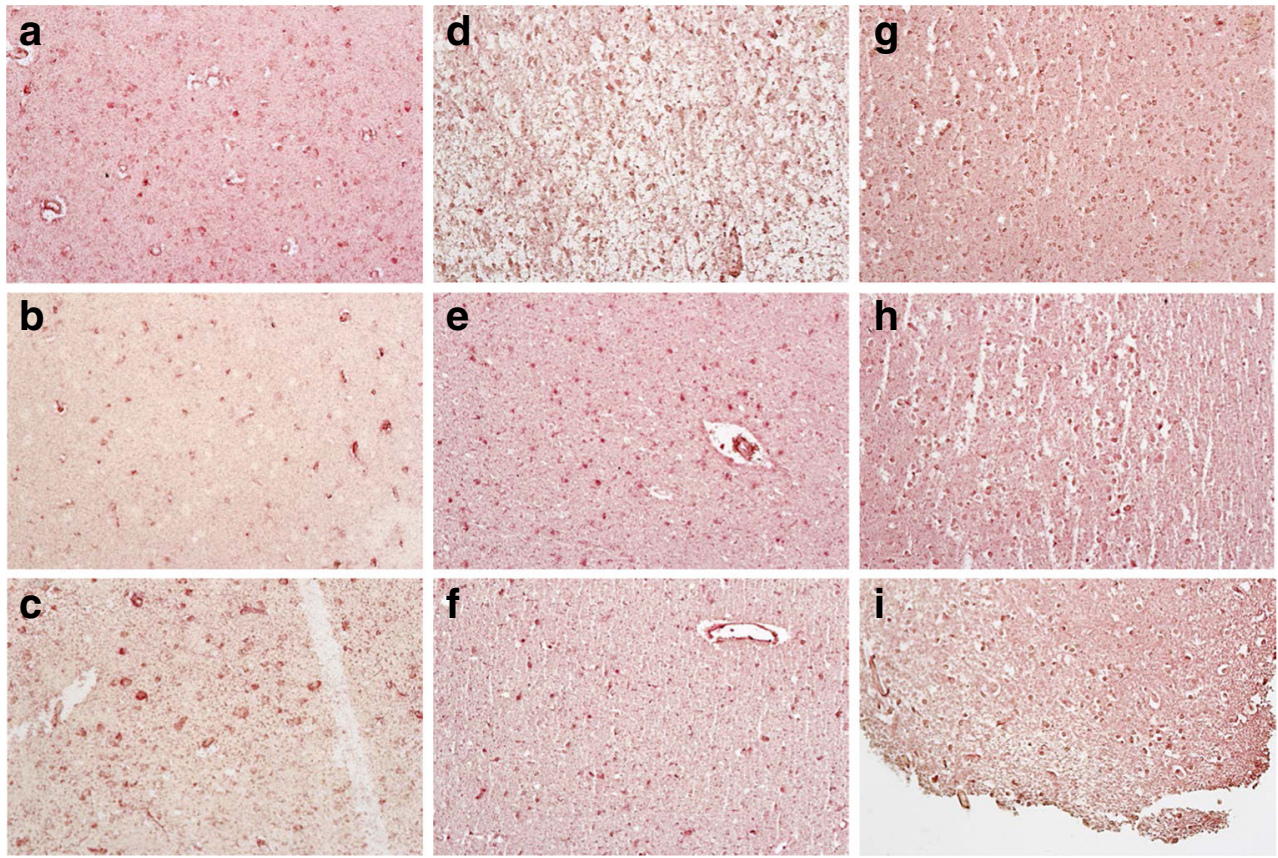

injury in the kidneys, lungs, and gastrointestinal tract and inflammation in the central nervous system (Bémeur and Butterworth 2013; Hilzendeger et al. 2014; Winterberg and Lu 2012). While all attempts are made to select the most appropriate controls, there are inherent limitations when

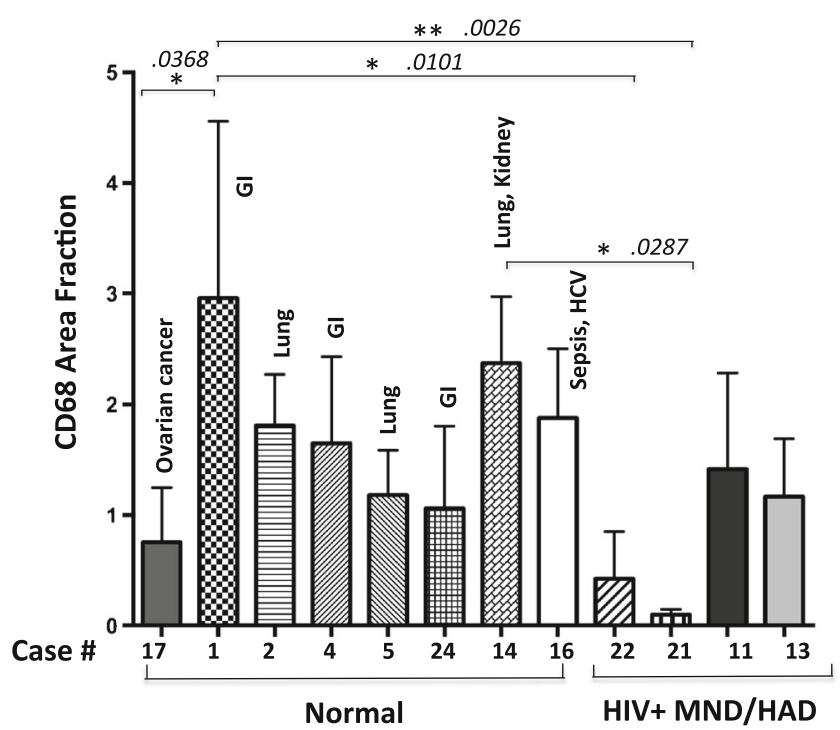

Fig. 9 CD68+ macrophages/microglia are readily detectable in normal control samples. ImageJ was used to quantify the area fraction of CD68 reactivity in three representative fields from each case. The mean and standard deviation and level of significance as determined by one-way ANOVA for normal controls and MND/HAD cases are shown. Category of clinical disorders (Table 2) at the time of death is indicated above the appropriate bars: GI gastrointestinal related, $H C V$ hepatitis $\mathrm{C}$ virus
Table 2 Clinical indications at the time of death

Patient Disorders at time of death

ID

Case 1 Upper GI bleed from ruptured esophageal varices; autoimmune hepatitis, ANA+ arthritis

Case 2 Tension pneumothorax most likely due to puncture of lung

Case 3 Multi-organ failure secondary to cardiogenic shock due to myocardial infarction in a failing heart with prior ischemic damage

Case 4 Hemorrhagic shock due to gastrointestinal bleeding from a large duodenal ulcer that destroyed a segment of the tastroduodenal artery; peptic ulcer disease

Case 5 Severe necrotizing tracheobronchitis and pneumonia due to community acquired-MRSA and beta hemolytic Streptococcus group B

Case 14 Severe mitral valvular disease with calcified bioprosthesis and calcific, infected emboli to multiple organs (consistent with strep pneumoniae); end-stage renal disease; bronchopneumonia

Case 15 Hepatitis C cirrhosis and end stage liver disease; staph aureus sepsis; prior IVDU

Case 16 Klebsiella sepsis with hepatic abscess, OLT for HCV cirrhosis, pulmonary hypertension, ECRP with sphincterotomy for biliary stones

Case 24 Scleroderma with CREST syndrome, GI hypomotility with large bowel obstruction, subtotal colectomy, erosive candidal esophagitis, and pneumoatosis intestinalis small intestine; pulmonary fibrosis, hypertension, and emphysema; arterionephrosclerosis; cachexia

Case 17 Ovarian cancer 
working with human clinical samples. Perhaps prescreening of normal controls for specific inflammatory markers could be used to select tissues with low levels of baseline activation. It should be noted that the samples in this study differed from those used in a prior report on OPN levels in the CSF (Brown et al. 2011).

Despite the possible limitations of the control samples used in this study, OPN was expressed at a significantly higher level in Iba-1/AIF-1+ microglia in both the HIV + ANI and $\mathrm{HIV}+\mathrm{MND} / \mathrm{HAD}$ groups suggesting that these cells are a major source of this proinflammatory cytokine in the brain. The HIV+ samples came from individuals who were on ART, yet elevated OPN levels appeared to be sustained over a period of many years. A recent study analyzing traditional markers of cellular activation using brain tissue from the frontal white matter and basal ganglia of HIV-infected individuals on ART with cognitive impairment, but no encephalitis, also found that inflammation persists in those without HIV encephalitis despite therapy (Tavazzi et al. 2014). Elevation of CD68+ macrophage/microglia in the absence of any evidence of local proliferation in autopsy samples from the hippocampus of individuals on highly active antiretroviral therapy compared to normal controls was seen in a study by Anthony et al. suggesting that these cells remain activated despite the effective suppression of HIV replication (Anthony et al. 2005). However, unlike our study, many of the samples were from individuals who abused drugs (and were $\mathrm{HCV}+$ ), a potential contributor to neuroinflammation (Anthony et al. 2005). Moreover, in the current study, 11 out of the 14 HIVinfected individuals, irrespective of detectable viral load in the plasma or CSF, were very immunosuppressed as determined by a CD4+ T cell level below 200 cells $/ \mathrm{ml}$ (Table 1 ). In $50 \%$ of the HIV+ cases, viral load was detected in the CSF suggesting the real possibility that persistent viral replication albeit at varying levels continued within the brain parenchyma.

Ionized $\mathrm{Ca}^{2+}$-binding adapter 1 (Iba1), which is thought to be the same gene product as allograft inflammatory factor-1 (AIF-1), is a $17-\mathrm{kDa}$ cytoplasmic protein that binds calcium whose function continues to be elucidated (Zhao et al. 2013). While Iba-1/AIF-1 is expressed constitutively, it can also be a marker of the cellular inflammatory response as it sits in a gene cluster with TNF- $\alpha$, TNF- $\beta$, and NF-KB in the MHC class III region (Zhao et al. 2013). Both ameboid and ramified microglia morphology was observed in most cases. Both types of microglia possess phagocytic activity and the ability to clear debris, while those with a ramified shape have been reported to contribute to efficient neurogenesis and the maintenance of synapses (Paolicelli et al. 2014; Perry and Teeling 2013). In the HIV+ MND/HAD group, microglia without any processes, which may be the ameboid type, often predominated suggesting that they are actively involved in phagocytosis and the clearance of dying cells. Alternatively, the absence of microglia with ramified morphology could indicate a functional deficit in the ability to conduct the high-level surveillance that is characteristic of microglia in the unimpaired brain (Nimmerjahn et al. 2005). In this regard, analyses of the Iba- 1 / OPN ratio showed that Iba-1 levels were significantly lower in all of the disease groups compared to the control.

This study is the first to show that OPN is also significantly elevated in activated microglia in ALS. The rod-like shape of microglia seen in this study has been recently reported in a rat model of brain injury (Ziebell et al. 2012) and was seen earlier in a model of experimental autoimmune encephalomyelitis or neuritis (Schluesener et al. 1998). Additional studies with a larger sample set and at different disease stages are required to determine whether the increase in OPN displays a temporal pattern. Interestingly, a recent study found that CD44, the receptor for OPN, is elevated in the SOD1 mouse model for familial ALS (Matsumoto et al. 2012). A connection between disease progression, deficits in executive function, and high levels of microglial activation has been found in ALS and is of relevance as this area of cognitive functioning is also compromised in HIV-infected individuals (Brettschneider et al. 2012a; Brettschneider et al. 2012b)

Our data revealed that in addition to microglia, neurons in HAND are a major source of OPN. Levels of OPN were highest in the HIV+ ANI group. This difference was not related to the overall levels of NeuN, as they were similar in the $\mathrm{HIV}+\mathrm{ANI}$ and HIV+ MND/HAD groups. A possible limitation is the small sample size as outliers could skew the results. In a prior study, we found that OPN levels in CSF were elevated in HIV-infected individuals with normal cognition and further increased with a higher level of impairment (Brown et al. 2011). Increases in OPN in the CSF, and specifically in neurons, have been reported in neurodegenerative disorders including multiple sclerosis, Alzheimer's and Parkinson's diseases, frontotemporal dementia, and in models of brain injury and stroke. In multiple sclerosis, elevations in CSF OPN are associated with worse disease outcomes and may be related to the ability of the cytokine to promote the survival of myelin reactive T cells (Hur et al. 2006; Vogt et al. 2004). In contrast, OPN perhaps acting through similar prosurvival pathways promotes wound healing and repair after ischemic injury (Chen et al. 2011; van Velthoven et al. 2011).

Our data provide support for the indirect pathway leading to neuronal damage and dysfunction in HIV-associated infection of the CNS. The trafficking of HIV-infected and activated macrophages, which are expressing OPN at a high level, suggests that the elevation of this cytokine in the brain would be an early event. Indeed, we found that HIV-infected individuals (without cognitive impairment) had higher levels of OPN than uninfected controls and those with MS (Brown et al. 2011). Inflammation in the brain is required for repair and normal homeostatic clearance activity. However, when inflammation is sustained over a long period of time, and at excessive levels, 
over activation of immune and CNS cells occur that leads, by mechanisms that remain to be fully elucidated, to functional dysregulation in which biological processes fail to behave in a normal fashion leading to cellular degeneration and death. Indeed, microglia play key roles in regulating neuronal cell death, neurogenesis, and synaptic interactions. Crosstalk through fractalkine receptor- and CD200 receptor-ligand interactions between neurons and microglia serve to regulate the inflammatory response and functional activity of these cells (Katsumoto et al. 2014).

Western blot analyses on brain cell lysates and ELISA measurements on CSF on samples from individuals with HAND revealed significant elevation in OPN that trended with the increase in cognitive impairment (Brown et al. 2011). This current study now demonstrates that the source of OPN is multifactorial coming from astrocytes, resident macrophage/microglia, and neurons. This finding expands our thinking about the possible autocrine and paracrine feedback mechanisms that can operate in the brain parenchyma to sustain OPN levels at this site. Moreover, astrocytes, microglia, and neurons all express the receptors for OPN, which include specific integrins and variants of CD44 (Ailane et al. 2013; Akiyama et al. 1993). OPN is a proinflammatory cytokine of the innate immune system that primes several downstream factors in the inflammatory cascade. Studies investigating the impact of OPN on the function of cells of the CNS in the context of HIV infection will allow us to discern whether downregulation of OPN would provide any therapeutic benefit.

\section{Materials and methods}

Patient samples and selection criteria

The study protocol was approved by the Johns Hopkins Institutional Review Board. Tissue sections prepared from the occipital lobe were obtained from the National NeuroAIDS Tissue Consortium (NNTC). This region of the brain has been previously shown to have abundant HIV infection and pathology. Cytomegalovirus encephalitis, toxoplasmosis (active and healed), aseptic leptomeningitis, bacterial leptomeningitis, lymphoma, contusions, focal infarcts, anoxic/ischemic damage, and tuberculosis were all excluded. In addition, for all past and current substance-induced major depressive disorder, cannabis, cocaine, opiate, and methadone use were excluded. Toxicology reports were examined and drug abuse and dependence were also excluded. All races as well as medically prescribed drugs were allowed. The revised American Academy of Neurology criteria were used to classify individuals into three groups based on cognitive function: asymptomatic neurocognitive impairment (ANI), HIVassociated mild neurocognitive disorder (MND), and HIV- associated dementia (HAD) (Antinori et al. 2007). Query of the NNTC database suggested that neurocognitive diagnosis consistent at the last two visits prior to death and the combination of MND and HAD subjects would yield the required number of cases. There were four groups examined: (1) HIVuninfected normal controls (normal, $n=9$ ); (2) neurocognitive disorder other, amyotrophic lateral sclerosis (ALS, $n=5)$; (3) HIV-infected asymptomatic neurocognitive impairment (ANI, $n=5$ ); and (4) HIV-infected minor neurocognitive disorder/ HIV-associated dementia (MND/HAD, $n=9)$. Case number 10 was included in the MND/HAD group based on the detection of HIV encephalitis (HIVE) on neuropathological examination.

\section{Immunohistochemical staining}

Paraffin-embedded sections were heated for $10 \mathrm{~min}$ at $60{ }^{\circ} \mathrm{C}$, then immersed in histoclear (Electron Microscopy Sciences) twice for $10 \mathrm{~min}$ each, followed by a 100, 95, and $70 \%$ ethanol gradient for a total of $10 \mathrm{~min}$ each. Slides were rinsed in $1 \times$ Tris-buffered saline (TBS) buffer (20 mM Tris, $13.8 \mathrm{mM}$ $\mathrm{NaCl}, \mathrm{pH}$ 7.4) and then treated with proteinase $\mathrm{K}$ solution (IHC World) for $20 \mathrm{~min}$ at $37^{\circ} \mathrm{C}$ in a humidified chamber. Depending on the antibody, slides were immersed in antigen retrieval buffer (10 mM Tris, $1 \mathrm{mM}$ EDTA, $0.05 \%$ Tween-20, $\mathrm{pH} 9.0$ ) or citric buffer ( $10 \mathrm{mM}$ sodium citrate, $0.05 \%$ Tween$20, \mathrm{pH} 6.0$ ) and placed in a steamer for $45 \mathrm{~min}$. Slides were incubated for $1 \mathrm{~h}$ in $10 \%$ goat serum/TBS followed by incubation with 1:100 dilution of rabbit anti-NeuN antibody (ABN78, Millipore), rabbit anti-GFAP (AB5804, Millipore), 1:50 rabbit anti-AIF-1 (HPA049234, SIGMA-Aldrich), and 1:50 rabbit anti-CD68 (bs-1432R, Bioss) at $4{ }^{\circ} \mathrm{C}$ overnight. The slides were rinsed thoroughly in $1 \times$ TBS followed by incubation in 1:500 dilution of goat anti-rabbit-alkaline phosphatase antibody (7054, Cell Signaling) at room temperature for $1 \mathrm{~h}$. Slides were rinsed in $1 \times$ TBS and developed with Permanent Fast-Red Quanto as directed by the manufacturer (Thermo Scientific) for 10-15 min at room temperature. The slides were rinsed in $1 \times$ TBS and treated with $3 \%$ hydrogen peroxide for 5-10 min and rinsed then incubated with 1:100 of mouse anti-OPN (MAB194P, Maine Biotechnology) for $1 \mathrm{~h}$ at room temp. Slides were rinsed in $1 \times$ TBS then incubated with 1:1500 dilution of anti-mouse HRP (7076S, Cell Signaling) for $1 \mathrm{~h}$ at room temperature. Slides were developed with DAB peroxidase substrate kit (Vector) for $2 \mathrm{~min}$, dehydrated, and mounted in Cytoseal 60 (Thermo Scientific).

Data and statistical analyses

Ten or twenty images from all four quadrants of the slides were taken at $\times 20$ (Zeiss Axio Observer A1 inverted microscope). The total area quantified was kept constant for each section analyzed. Adjustment of the image brightness, 
contrast, and sharpness was performed with Adobe Photoshop 5.5 using the same settings for each image. The images were saved as a new jpg file at a resolution of $300 \mathrm{dpi}$. The images were then opened in ImageJ and the colors split (Fig. 5) and pixel intensity quantified using the threshold function to select the outline of the cells of interest and measurement function to determine the area fraction of intensity. The total area examined for each sample remained constant. The area fractions determined from ImageJ were imported into GraphPad Prism 6 and analyzed by one-way ANOVA and Tukey's correction for multiple comparisons with significance of $p<0.05$.

Acknowledgments We acknowledge the technical assistance of Agasou Rameau and Shawnita Cureton. This work could not have been done without the resources provided by the US National NeuroAIDS Tissue Consortium through the following grants: Manhattan HIV Brain Bank: U01MH083501, R24MH59724; Texas NeuroAIDS Research Center U01MH083507, R24 NS45491; National Neurological AIDS Bank 5U01MH083500, NS 38841; California NeuroAIDS Tissue Network U01MH083506, R24MH59745; and Statistics and Data Coordinating Center U01MH083545, N01MH32002. This study was also made possible through funding from the US National Institutes of Mental Health grant R21 MH095646 awarded to A.B..

Conflict of interest The authors Katie Silva, Calixto Hope-Lucas, Tyesha White, Tai Hairston, Tatenda Rameau, and Amanda Brown declare they have no conflicts of interest to disclose.

Open Access This article is distributed under the terms of the Creative Commons Attribution License which permits any use, distribution, and reproduction in any medium, provided the original author(s) and the source are credited.

\section{References}

Ailane S, Long P, Jenner P, Rose S (2013) Expression of integrin and CD44 receptors recognising osteopontin in the normal and LPSlesioned rat substantia nigra. Eur J Neurosci 38:2468-2476

Akiyama H, Tooyama I, Kawamata T, Ikeda K, McGeer P (1993) Morphological diversities of CD44 positive astrocytes in the cerebral cortex of normal subjects and patients with Alzheimer's disease. Brain Res 632:249-259

Anthony IC, Ramage SN, Carnie FW, Simmonds P, Bell JE (2005) J Neuropathol Exp Neurol 64(6):529-536

Antinori A, Arendt G, Becker JT, Brew BJ, Byrd DA, Cherner M, Clifford DB, Cinque P, Epstein LG, Goodkin K, Gisslen M, Grant I, Heaton RK, Joseph J, Marder K, Marra CM, McArthur JC, Nunn M, Price RW, Pulliam L, Robertson KR, Sacktor N, Valcour V, Wojna VE (2007) Updated research nosology for HIV-associated neurocognitive disorders. Neurology 69:1789-1799

Bémeur C, Butterworth R (2013) Liver-brain proinflammatory signalling in acute liver failure: role in the pathogenesis of hepatic encephalopathy and brain edema. Metab Brain Dis 28:145-150

Braitch M, Nunan R, Niepel G, Edwards LJ, Constantinescu CS (2008) Increased osteopontin levels in the cerebrospinal fluid of patients with multiple sclerosis. Arch Neurol 65:633-635

Brenchley J, Price D, Schacker T, Asher T, Silvestri G, Rao S, Kazzaz Z, Bornstein E, Lambotte O, Altmann D, Blazar B, Rodriguez B, Teixeira-Johnson L, Landay A, Martin J, Hecht F, Picker L,
Lederman M, Deeks S, Douek D (2006) Microbial translocation is a cause of systemic immune activation in chronic HIV infection. Nat Med 12:1365-1371

Brettschneider J, Libon DJ, Toledo JB, Xie SX, McCluskey L, Elman L, Geser F, Lee VM, Grossman M, Trojanowski JQ (2012a) Microglial activation and TDP-43 pathology correlate with executive dysfunction in amyotrophic lateral sclerosis. Acta Neuropathol 123:395407

Brettschneider J, Toledo JB, Van Deerlin VM, Elman L, McCluskey L, Lee VM, Trojanowski JQ (2012b) Microglial activation correlates with disease progression and upper motor neuron clinical symptoms in amyotrophic lateral sclerosis. PLoS One 7:e39216

Brites D, Vaz AR (2014) Microglia centered pathogenesis in ALS: insights in cell interconnectivity. Front Cell Neurosci 8. doi:10.3389/ fncel.2014.00117

Brown A, Islam T, Adams R, Nerle S, Kamara M, Eger C, Marder K, Cohen B, Schifitto G, McArthur J, Sacktor N, Pardo CA (2011) Osteopontin enhances HIV replication and is increased in the brain and cerebrospinal fluid of HIV-infected individuals. J Neurovirol 17: 382-392

Budka H, Costanzi G, Cristina S, Lechi A, Parravicini C, Trabattoni R, Vago L (1987) Brain pathology induced by infection with the human immunodeficiency virus (HIV). A histological, immunocytochemical, and electron microscopical study of 100 autopsy cases. Acta Neuropathol 75:185

Burdo TH, Wood MR, Fox HS (2007) Osteopontin prevents monocyte recirculation and apoptosis. J Leukoc Biol 81:1504-1511

Burdo TH, Ellis RJ, Fox HS (2008) Osteopontin is increased in HIVassociated dementia. J Infect Dis 198:715-722

Burdo TH, Lo J, Abbara S, Wei J, DeLelys ME, Preffer F, Rosenberg ES, Williams KC, Grinspoon S (2011) Soluble CD163, a novel marker of activated macrophages, is elevated and associated with noncalcified coronary plaque in HIV-infected patients. J Infect Dis 204:1227-1236

Chabas D, Baranzini SE, Mitchell D, Bernard CC, Rittling SR, Denhardt DT, Sobel RA, Lock C, Karpuj M, Pedotti R, Heller R, Oksenberg JR, Steinman L (2001) The influence of the proinflammatory cytokine, osteopontin, on autoimmune demyelinating disease. Science 294:1731-1735

Chen W, Ma Q, Suzuki H, Hartman R, Tang J, Zhang JH (2011) Osteopontin reduced hypoxia-ischemia neonatal brain injury by suppression of apoptosis in a rat pup model. Stroke 42:764-769

Clements JE, Babas T, Mankowski JL, Suryanarayana K, Piatak M, Tarwater PM, Lifson JD, Zink MC (2002) The central nervous system as a reservoir for simian immunodeficiency virus (SIV): steadystate levels of SIV DNA in brain from acute through asymptomatic infection. J Infect Dis 186

Comabella M, Pericot I, Goertsches R, Nos C, Castillo M, Blas Navarro J, Rio J, Montalban X (2005) Plasma osteopontin levels in multiple sclerosis. J Neuroimmunol 158:231-239

Comi C, Carecchio M, Chiocchetti A, Nicola S, Galimberti D, Fenoglio C, Cappellano G, Monaco F, Scarpini E, Dianzani U (2010) Osteopontin is increased in the cerebrospinal fluid of patients with Alzheimer's disease and its levels correlate with cognitive decline. J Alzheimers Dis 19:1143-1148

Davis L, Hjelle B, Miller V, Palmer D, Llewellyn A, Merlin T, Young S, Mills R, Wachsman W, Wiley CA (1992) Early viral brain invasion in iatrogenic human immunodeficiency virus infection. Neurology 42:1736-1739

Deeks SG, Lewin SR, Havlir DV (2013) The end of AIDS: HIV infection as a chronic disease. Lancet 382:1525-1533

Eger C, Cirelli K, Budiaman J, Brown A (2014) Noncontiguous protein interaction domains in osteopontin contribute to enhance HIV-1 replication. J Hum Virol Retrovirol 1. doi:10.15406/jhvrv.2014.01. 00003 
Esiri MM, McGee JO (1986) Monoclonal antibody to macrophages $(\mathrm{EMB} / 11)$ labels macrophages and microglial cells in human brain. J Clin Pathol 39:615-621

Fischer-Smith T, Croul S, Adeniyi A, Rybicka K, Morgello S, Khalili K, Rappaport J (2004) Macrophage/microglial accumulation and proliferating cell nuclear antigen expression in the central nervous system in human immunodeficiency virus encephalopathy. Am J Pathol 164:2089-2099

Glass J, Fedor H, Wesselingh S, McArthur J (1995) Immunocytochemical quantitation of human immunodeficiency virus in the brain: correlations with dementia. Ann Neurol 38:755-762

Heaton RK, Clifford DB, Franklin DR Jr, Woods SP, Ake C, Vaida F, Ellis RJ, Letendre SL, Marcotte TD, Atkinson JH, Rivera-Mindt M, Vigil OR, Taylor MJ, Collier AC, Marra CM, Gelman BB, McArthur JC, Morgello S, Simpson DM, McCutchan JA, Abramson I, Gamst A, Fennema-Notestine C, Jernigan TL, Wong J, Grant I, Group C (2010) HIV-associated neurocognitive disorders persist in the era of potent antiretroviral therapy: CHARTER Study. Neurology 75:2087-2096

Heaton RK, Franklin DR, Ellis RJ, McCutchan JA, Letendre SL, LeBlanc S, Corkran DB, Woods SP, Collier AC, Marra CM, Morgello S, Mindt MR, Taylor MJ, Marcotte TD, Atkinson JH, Wolfson T, Gelman BB, McArthur JC, Simpson DM, Abramson I, Gamst A, Fennema-Notestine C, Jernigan TL, Wong J, Grant I (2011) HIVassociated neurocognitive disorder before and during the era of combination antiretroviral therapy: differences in rates, nature and predictors. J Neurovirol 17:3-16

Hilzendeger A, Shenoy V, Raizada M, Katovich M (2014) Neuroinflammation in pulmonary hypertension: concept, facts, and relevance. Curr Hypertens Rep 16. doi:10.1007/s11906-014-0469-1

Hur EM, Youssef S, Haws ME, Zhang SY, Sobel RA, Steinman L (2006) Osteopontin-induced relapse and progression of autoimmune brain disease through enhanced survival of activated T cells. Nat Immunol 8:74-83

Iczkiewicz J, Jackson MJ, Smith LA, Rose S, Jenner P (2006) Osteopontin expression in substantia nigra in MPTP-treated primates and in Parkinson's disease. Brain Res 1118:239-250

Jansson M, Panoutsakopoulou V, Baker J, Klein L, Cantor H (2002) Cutting edge: attenuated experimental autoimmune enchephalomyelitis in eta-1/osteopontin-deficient mice. J Immunol 168:2096-2099

Katsumoto A, Lu H, Miranda AS, Ransohoff RM (2014) Ontogeny and functions of central nervous system macrophages. J Immunol 193: 2615-2621

Koenig S, Gendelman HE, Orenstein JM, Dal Canto MC, Pezeshkpour GH, Yungbluth M, Janotta F, Aksamit A, Martin MA, Fauci AS (1986) Detection of AIDS virus in macrophages in brain tissue from AIDS patients with encephalopathy. Science 233:1089-1093

Kraft-Terry SDSA, Buch S, Gendelman HE (2010) HIV-1 neuroimmunity in the era of antiretroviral therapy. Neurobiol Dis 37:542-548

Lucas C-H, Calvez M, Brown A (2014) Altered subcellular localization of the NeuN/Rbofox3 RNA splicing factor in HIV-associated neurocognitive disorders (HAND). Neurosci Lett 558:97-102

Maetzler W, Berg D, Schalamberidze N, Melms A, Schott K, Mueller JC (2007) Osteopontin is elevated in Parkinson's disease and its absence leads to reduced neurodegeneration in the MPTP model. Neurobiol Dis 25:473-482

Matsumoto T, Imagama S, Hirano K, Ohgomori T, Natori T, Kobayashi K, Muramoto A, Ishiguro N, Kadomatsu K (2012) CD44 expression in astrocytes and microglia is associated with ALS progression in a mouse model. Neurosci Lett 520

Mattsson N, Rüetschi U, Pijnenburg YA, Blankenstein MA, Podust VN, Li S, Fagerberg I, Rosengren L, Blennow K, Zetterberg H (2008)
Novel cerebrospinal fluid biomarkers of axonal degeneration in frontotemporal dementia. Mol Med Rep 757:757-761

McArthur J, Steiner J, Sacktor N, Nath A (2010) Human immunodeficiency virus-associated neurocognitive disorders: mind the gap. Ann Neurol 67:699-714

Meulendyke K, Queen S, Engle E, Shirk E, Liu J, Steiner J, Nath A, Tarwater P, Graham D, Mankowski J, Zink M (2014) Combination fluconazole/paroxetine treatment is neuroprotective despite ongoing neuroinflammation and viral replication in an SIV model of HIV neurological disease. J Neurovirol

Nimmerjahn A, Kirchhoff F, Helmchen F (2005) Resting microglial cells are highly dynamic surveillants of brain parenchyma in vivo. Science 308:1314-1318

Paolicelli R, Kanchan B, Tremblay M-E (2014) Fractalkine regulation of microglial physiology and consequences on the brain and behavior. Fron Cell Neuro 8. doi:10.3389/fncel.2014.00129

Perry V, Teeling J (2013) Microglia and macrophages of the central nervous system: the contribution of microglia priming and systemic inflammation to chronic neurodegeneration. Semin Immunopathol 35:601-612

Roberts ES, Zandonatti MA, Watry DD, Madden LJ, Henriksen SJ, Taffe MA, Fox HS (2003) Induction of pathogenic sets of genes in macrophages and neurons in NeuroAIDS. Am J Pathol 162:2041-2057

Schluesener HJ, Seid K, Kretzschmar J, Meyermann R (1998) Allograftinflammatory factor-1 in rat experimental autoimmune encephalomyelitis, neuritis, and uveitis: expression by activated macrophages and microglial cells. Glia 24:244-251

Spudich S, Gisslen M, Hagberg L, Lee E, Liegler T, Brew B, Fuchs D, Tambussi G, Cinque P, Hecht FM, Price RW (2011) Central nervous system immune activation characterizes primary human immunodeficiency virus 1 infection even in participants with minimal cerebrospinal fluid viral burden. J Infec Dis 204:753-760

Tavazzi E, Morrison D, Sullivan P, Morgello S, Fischer T (2014) Brain inflammation is a common feature of HIV-infected patients without HIV encephalitis or productive brain infection. Curr HIV Res 12:97110

Tovar-y-Romo LB, Bumpus NN, Pomerantz D, Avery LB, Sacktor N, McArthur JC, Haughey NJ (2012) Dendritic spine injury induced by the 8-hydroxy metabolite of efavirenz. J Pharmacol Exp Ther 343: 696-703

van Velthoven CT, Heijnen CJ, van Bel F, Kavelaars A (2011) Osteopontin enhances endogenous repair after neonatal hypoxicischemic brain injury. Stroke 42:2294-2301

Vázquez-Santiago FJ, Noel RJJ, Porter JT, Rivera-Amill V (2014) Glutamate metabolism and HIV-associated neurocognitive disorders. J Neurovirol 20:315-331

Vogt MH, Floris S, Killestein J, Knol DL, Smits M, Barkhof F, Polman CH, Nagelkerken L (2004) Osteopontin levels and increased disease activity in relapsing remitting multiple sclerosis patients. $\mathrm{J}$ Neuroimmunol 155:155-160

Winterberg P, Lu C (2012) Acute kidney injury: the beginning of the end of the dark ages. Am J Med Sci 344:318-325

Wirths O, Breyhan H, Marcello A, Cotel MC, Brück W, Bayer TA (2010) Inflammatory changes are tightly associated with neurodegeneration in the brain and spinal cord of the APP/PS1KI mouse model of Alzheimer's disease. Neurobiol Aging 31:747-757

Zhao YY, Yan DJ, Zhen ZW (2013) Role of AIF-1 in the regulation of inflammatory activation and diverse disease processes. Cell Immunol 284:75-83

Ziebell JM, Taylor SE, Cao T, Harrison JL, Lifshitz J (2012) Rod microglia: elongation, alignment, and coupling to form trains across the somatosensory cortex after experimental diffuse brain injury. J Neuroinflam 9. doi:10.1186/1742-2094-9-247 